\title{
Hypercalcemia in Advanced Prostatic Carcinoma: A Rare Event
}

\author{
Mary Mathews ${ }^{1, *}$, Abhishek Kumar², Nelly Awkar², Deepak Dhaliwal ${ }^{1}$, Michael Maroules² \\ ${ }^{1}$ Department of Internal Medicine, St. Joseph’s Regional Medical Center, New York Medical College \\ ${ }^{2}$ Division of Hematology Oncology, Department of Internal Medicine, St. Joseph’s Regional Medical Center \\ *Corresponding author: mary.mathews@live.ca
}

Received January 09, 2015; Revised January 24, 2015; Accepted January 29, 2015

\begin{abstract}
Prostate cancer is typically associated with metastatic osteoblastic lesions, hypocalcemia and hypophosphatemia. Hypercalcemia is a rarely encountered phenomenon i.e. 1-2\% in prostate cancer, although it is well associated with other malignant cancers involving breast, lung, head, neck and multiple myelomas. We present the case of a 63 year old Mexican man with history of advanced prostatic adenocarcinoma and trans-urethral resection of prostate presented to ER with nausea, vomiting, polydipsia and altered mental status. Clinically, he appeared dehydrated and pale, with no other remarkable findings. Laboratory findings indicated severe hypercalcemia with calcium-16.1 mg/dL, with imaging studies revealing extensive metastatic osteoblastic lesions. Our patient was treated with aggressive hydration, calcitonin and pamidronic acid, his clinical status improved with complete resolution of hypercalcemia. We have discussed the possible underlying mechanisms and their interplay involved in the presentation of hypercalcemia in prostate cancer, and that its occurence is unlikely due to skeletal metastases.
\end{abstract}

\section{Keywords: prostate cancer, hypercalcemia, skeletal metastasis, osteoblastic lesions}

Cite This Article: Mary Mathews, Abhishek Kumar, Nelly Awkar, Deepak Dhaliwal, and Michael Maroules, "Hypercalcemia in Advanced Prostatic Carcinoma: A Rare Event." American Journal of Medical Case Reports, vol. 3, no. 3 (2015): 64-65. doi: 10.12691/ajmcr-3-3-3.

\section{Case Report}

A 63 year old Mexican man with history of advanced prostatic adenocarcinoma and trans-urethral resection of prostate presented to ER with nausea, vomiting, polydipsia and altered mental status. The patient had previously received multiple lines of therapies including leuprolide, pelvic radiation therapy, docetaxel, cabazitaxel and abiraterone. He was medically castrated secondary to leuprolide therapy, with a testosterone level level 1.20 ng/dL. Clinically, he appeared dehydrated and pale, with no other remarkable findings. Laboratory findings indicated severe hypercalcemia with calcium-16.1 mg/dL, serum alkaline phosphatase $-251 \mathrm{IU} / \mathrm{L}, \mathrm{PSA}>149 \mathrm{ng} / \mathrm{mL}$, hemoglobin-7.6 g/dL and platelets-61,000, BUN-30 $\mathrm{mg} / \mathrm{dL}$ and creatinine- $2.44 \mathrm{mg} / \mathrm{dL}$, intact parathyroid hormone(PTH) of $10.5 \mathrm{PG} / \mathrm{mL}$ and PTH related peptide (PTHrp) of $0.74 \mathrm{pmol} / \mathrm{L}$ (normal $<2.0 \mathrm{pmol} / \mathrm{L}$ ).Despite the presence of severe hypercalcemia, he did not develop any cardiac arrythmias. He underwent a PET scan (Figure 1A) and bone scan (Figure 1B) revealing extensive metastatic osteoblastic lesions. His bone marrow biopsy was indicative of metastatic carcinoma of prostatic origin, resulting in severe anemia and thrombocytopenia. The patient was treated with aggressive hydration, calcitonin and pamidronic acid. The patient's mental status improved with resolving hypercalcemia and kidney functions, he was discharged on enzalutamide, leuprolide and zometa. Our patient had followed up two weeks after discharge, his improvement in clinical status was maintained and there was complete resolution of the hypercalcemia with calcium of $9.5 \mathrm{mg} / \mathrm{dL}$.

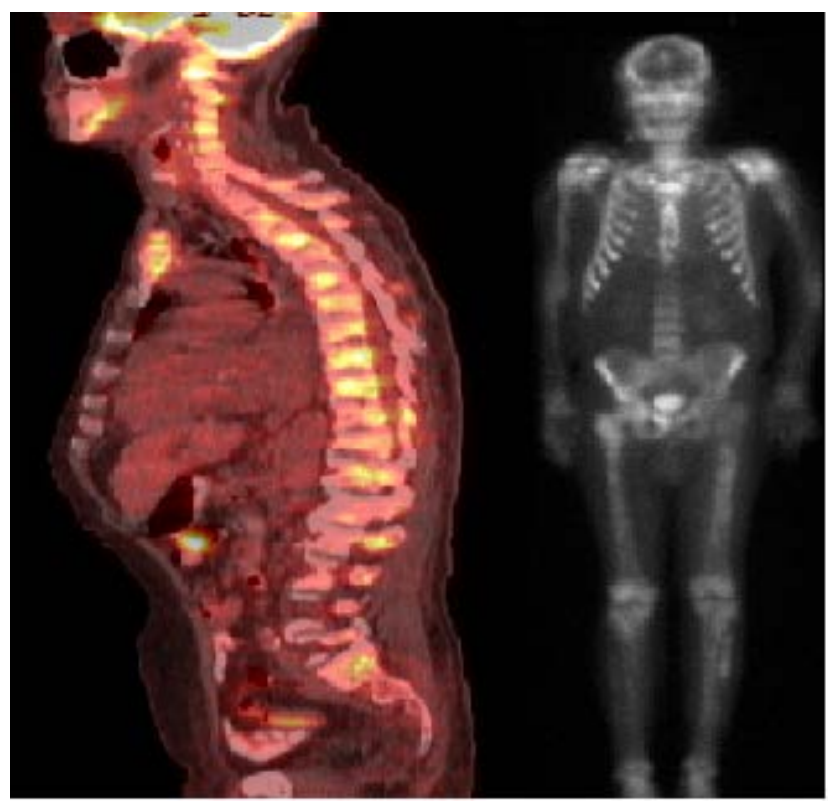

Figure 1. PET scan (A) and Bone scan (B), demonstrating extensive metastaic osteoblastic lesions 


\section{Discussion}

Prostate cancer is typically associated with metastatic osteoblastic lesions, hypocalcemia and hypophosphatemia. $[1,2,3]$ Hypercalcemia is rarely encountered in prostate cancer, although it is well associated with other malignant cancers involving breast, lung, head, neck and multiple myelomas. [1,2] Despite affinity of prostate cancer towards skeletal metastases, only $1-2 \%$ of patients present with hypercalcemia. $[3,5,6]$ Hypercalcemia may indicate a tumor recurrence or a preterminal event, including increased risk of detrimental skeletal events and death. [1,3] Many different mechanisms of malignancy associated hypercalcemia have been proposed, such as humoral mechanisms (PTH or PTH like peptides, prostaglandins etc.), direct-release mechanism (osteolytic stimulation via neoplastic cells), epidermal growth factors (EGF), transforming growth factors (TGF) and lastly inherent tumor mosaicism. [3,4] Laboratory results on our patient ruled out hyperparathyroidism and PTHrp related hypercalcemia. Our Imaging studies indicated a osteoblastic nature of lesions favoring a positive calcium balance, suggesting a different underlying pathophysiology of mineral metabolism in these patients [1,4].

We postulate that the presence of hypercalcemia is unlikely solely due to skeletal metastases in our patient, but is the interplay of osteoclastic bone resorption via EGF, TGF and underlying tumor mosaicism.
In conclusion, we believe that independent and synergistic roles between direct stimulation of osteoclastic activity (via neoplastic cells), growth factors (like EGF, TGF) as well as underlying tumor mosaicism should be considered by physicians in patients presenting with unexplained hypercalcemia in prostatic carcinoma, it is not merely an extension of skeletal metastases.

\section{References}

[1] Tucci, M., et al. Prognostic significance of disordered calcium metabolism in hormone-refractory prostate cancer patients with metastatic bone disease. Prostate cancer and prostatic diseases. 2008; 12 (1):94-99.

[2] Raskin, Philip, Craig J. McClain, and Thomas A. Medsger. Hypocalcemia associated with metastatic bone disease: a retrospective study. Archives of internal medicine. 1973; 132(4): 539-543.

[3] Avashia, Jay H., et al. Metastatic carcinoma of the prostate with hypercalcemia. Cleveland Clinic journal of medicine. 1990; 57. 7: 636-638.

[4] Olsson, Arne M., and Gösta Jönsson. Advanced cancer of the prostate combined with hypercalcaemia. Scandinavian journal of urology and nephrology. 1977; 11(3): 293-296.

[5] Wu, Ching-Heng, et al. Hypercalcemia in prostate cancer with positive neuron-specific enolase stain. Renal failure. 2004; 26(3): 325-327.

[6] Mahadevia, Panna S., et al. Hypercalcemia in prostatic carcinoma: report of eight cases. Archives of internal medicine. 1983; 143(7): 1339-1342. 